Journal of Theoretical and Applied Mechanics, Sofia, 2016, vol. 46, No. 3, pp. 35-64

\title{
EFFECTS OF DISCRETE DAMPING ON THE DYNAMIC BEHAVIOUR OF ROTATING SHAFT THROUGH EXTENDED LAGRANGIAN FORMULATION
}

\author{
VIKAS RASTOGi \\ Department of Mechanical, Production \& Industrial and Automotive Engineering, \\ Delhi Technological University, Delhi-110042, India, \\ e-mail : rastogivikas@yahoo.com
}

[Received 26 December 2014. Accepted 20 June 2016]

\begin{abstract}
The main focus of the paper is touted as effects of discrete damping on the dynamic analysis of rotating shaft. The whole analysis is being carried out through extended Lagrangian formulation for a discrete - continuous system. The variation formulation for this system is possible, considering the continuous system as one-dimensional. The generalized formulation for one dimensional continuous rotary shaft with discrete external damper has been obtained through principle of variation. Using this extended formulation, the invariance of umbra-Lagrangian density through extended Noether's theorem is achieved. Rayleigh beam model is used to model the shaft. Amplitude equation of rotor is obtained theoretically and validated through simulation results. The simulation results reveal the important phenomena of limiting dynamics of the rotor shaft, which is due to an imbalance of material damping and stiffness of the rotor shaft. The regenerative energy in the rotor shaft, induced due to elasticity/stiffness of the rotor shaft, is dissipated partially through the inspan discrete damper and also through the dissipative coupling between drive and the rotor shaft. In such cases, the shaft speed will not increase with increase in excitation frequency of the rotor but the slip between the drive and the shaft increases due to loading of drive.

KEY wORDS: Umbra-Lagrangian density, extended Noether's theorem, dynamic analysis, in-span discrete damping.
\end{abstract}

\section{Introduction}

It has been seen in most of the cases of continuous systems that employed methods of Lagrange and Hamilton are basically obtained through the

\footnotetext{
* This paper is a tribute to late Prof. Amalendu Mukherjee, who has formulated the concept of umbra-Lagrangian; and Prof. Anirvan Dasgupta, Professor, Mechanical Department, IIT, Kharagpur for their valuable suggestions throughout the course of this study.
} 
variation principle. There are several direct methods available in literature, which approximate the continuous system. In such approach, the system is discretized into finite or discrete particles and equations are obtained, which are finally approached to the continuous limits. However, such methods do not provide generality to the solution. The general relationship between oneparameter continuous symmetry and conserved quantity, specially in field's theory has been achieved by Boyer [1]. Some direct method of associating conserved quantities of Lagrangian system with each dynamical symmetry group has been reported by Sarlet and Cantrijn [2]. Other symmetry aspects of twodimensional and three dimensional order Lagrangian and Hamiltonian formalism are studied by Katzin and Levine [3] and Damianou and Sophocleous [4]. However, such methods are employed only to a system of some specific configuration; and generality to the application in the interest of science and engineering are quite missing.

A new proposal of umbra-time was made by Mukherjee [5] to extend the scope of Lagrangian-Hamiltonian mechanics. A brief and promising commentary on such extension has been given by Brown [6]. The detailed theory and applications of an extended Lagrangian-Hamiltonian mechanics are presented in various references [7, 8, 10 and 11]. Further, Mukherjee et. al. [9] has applied the extended Lagrangian- Hamiltonian mechanics for a general class of continuous systems, which incorporated gyroscopic coupling and non-conservative fields, including internal and external damping. Both damping were distributed homogeneously throughout the system. However, the methodology and case discussed in ref. [9] did not include the concept of discrete-continuous hybrid system, which is formulated in this research work.

Rotor dynamics is a subject of interest from the middle of $20^{\text {th }}$ Century $[12,13]$. This area is consistently in demand and has drawn attentions throughout the globe due to different effects and phenomena [14]. It is a well known fact, that both internal and external damping is significant for rotating shafts. The study of spinning shaft with internal damping was first investigated by Kimball [15]. Although Kimball's study did not specify the type of damping, he stated that internal friction due to bending in a spinning shaft contributes to shaft whirling above the first critical speed. Dimentberg [16] and Gunter [17] illustrated the concepts of the first critical speed due to internal viscous damping, which ultimately led to instability in the rotating shaft. Ehrich [18] studied the stability relationship between internal and external damping and noted that the rotational speed, at which the rotor becomes unstable, is governed by the ratio of internal and external damping. Some interesting results on modelling of internal damping in rotors were reported in the papers of Cran- 
dall [19], Zorzi and Nelson [20], Melanson and Zu [21], Genta [22], and Vance and Lee. [23]. In some of the studies, the instabilities resulting from internal damping are characterized. It is noteworthy that damping inform of material damping contribute the circulatory effects. Internal damping in such cases has a destabilizing influence on the rotor system. The discrete-continuous modelling of a rotor system was presented by Szoic [24]. In this paper, dynamical investigations of a rotor shaft system were performed by means of discrete continuous mechanical models. In such models, a rotating cylindrical shaft was represented as continuous system, whereas bearings were assumed as discrete elements. In another study by Jivkov and Zahariev [25], the stability and non-stationary vibrations of rotor-shaft in elastic-viscous field have been investigated through Bogoliubov-Mitropolski's asymptotical method. Further, Krenk [26] has demonstrated the complex mode analysis of cable and beam problems with concentrated viscous dampers. In these problems, the dissipating effect of the damper is closely linked with the complex character of the modes.

The main focus of this paper includes the extension of LagrangianHamiltonian mechanics for a discrete-continuous hybrid system. In this extension, umbra-Lagrangian density is efficiently used to describe the motion of the system. The invariance of umbra-Lagrangian density (ULD) may be achieved by application of extended Noether's theorem over manifolds. The general formulation for dissipative effects with in-span concentrated discrete damper is developed through extended Lagrangian formulation. A case study of a rotating shaft with in-span discrete damper is analyzed, which shows a significant phenomenon of limiting dynamics of rotor, beyond threshold speed of instability. The effect of discrete external damping is examined and entrainment of critical whirling speeds at natural undamped modes is observed. A simulation rig is also created to animate and visualize the effect of in-span discrete damper on natural modes of rotating shaft.

\section{Methodology}

\subsection{Generalized formulation for one-dimensional discrete-con-} tinuous hybrid system through principle of variation

Generalized formulation for one-dimensional discrete-continuous hybrid system shaft may be achieved by defining the system over the closed domain $x_{0} \leq x \leq x_{1}$, as shown in Fig. 1, where $t_{1}$ and $t_{2}$ are considered as arbitrary times and $x$ is the distance of any material point of the system.

In this generalized formulation of examining the dissipative effects, various gyroscopic forces, rotary inertia, internal damping and in-span dissipation 

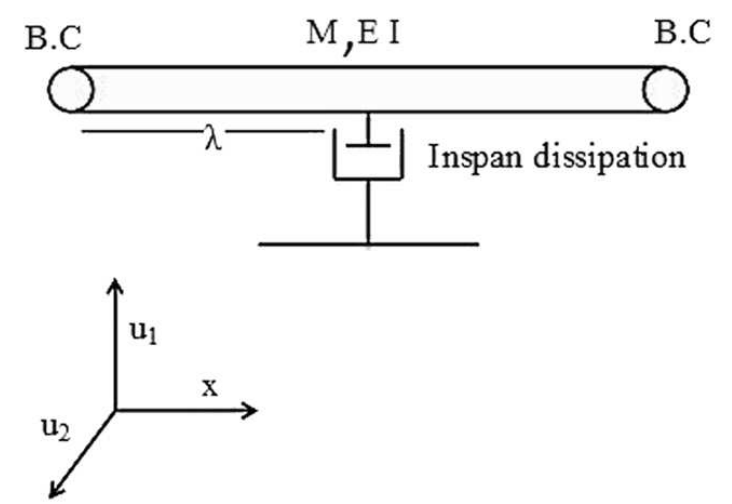

Fig. 1. Schematic diagram of a continuous shaft with uniform mass and constant rigidity with Inspan discrete damper

has been considered. It is assumed, that shaft is rotated at constant speed with $\omega$. Umbra-Lagrangian density may be obtained as:

(1)

$$
L=\left[\begin{array}{l}
\sum_{i=1}^{2}\left\{\begin{array}{l}
\frac{1}{2} \rho\left(\frac{\partial u_{i}(\eta, x)}{\partial \eta}\right)^{2}-\frac{1}{2} E I\left(\frac{\partial^{2} u_{i}(\eta, x)}{\partial x^{2}}\right)^{2} \\
+\frac{1}{2} I_{d}\left(\frac{\partial^{2} u_{i}(\eta, x)}{\partial \eta \partial x}\right)^{2}
\end{array}\right\} \\
-\omega I_{p}\left(\frac{\partial^{2} u_{1}(t, x)}{\partial t \partial x} \frac{\partial u_{2}(\eta, x)}{\partial x}-\frac{\partial^{2} u_{2}(t, x)}{\partial t \partial x} \frac{\partial u_{1}(\eta, x)}{\partial x}\right) \\
+\sum_{i=1}^{2} R_{a} \delta(x-\lambda)\left(\frac{\partial u_{i}(\eta, x)}{\partial \eta}\right)^{2} \\
-\mu_{i}\left\{\left(\frac{\partial^{3} u_{1}(t, x)}{\partial t \partial x^{2}}+\omega \frac{\partial^{2} u_{2}(t, x)}{\partial x^{2}}\right) \frac{\partial^{2} u_{1}(\eta, x)}{\partial x^{2}}\right. \\
\left.+\left(\frac{\partial^{3} u_{2}(t, x)}{\partial t \partial x^{2}}-\omega \frac{\partial^{2} u_{1}(t, x)}{\partial x^{2}}\right) \frac{\partial^{2} u_{2}(\eta, x)}{\partial x^{2}}\right\}
\end{array}\right] .
$$

In the above Eq. (1), $u_{i}()$ is the displacement coordinates of the rotor in real or umbra time, $\rho$ is the material density of the rotating shaft, $E I$ is the 
rigidity, $I_{d}$ is the rotary inertia, $I_{p}$ is the inertia of the rotating shaft through principle axis, $\omega$ is the constant angular velocity, $\mu_{i}$ is internal damping of the shaft, $R_{a}$ is the in-span dissipating element. In Eq. (1), fifth term is umbrapotential, due to In-span external dissipation.

Now, one may take umbra-Lagrangian density variation, which may be expressed as variations in the action integral in homotopic functions notated as $U_{i}(t, x, a)$ and $U_{i}(t, x, b)$, where $i=1,2$ :

$$
\delta I=\varepsilon \int_{t_{0}}^{t_{1}} \int_{x_{0}}^{x_{1}} \sum_{i=1}^{2}\left[\begin{array}{c}
\left\{\frac{\partial L}{\partial U_{i}(t, x, b)}-\frac{\partial}{\partial t} \frac{\partial L}{\partial \dot{U}_{i}(t, x, b)}\right. \\
\left.-\frac{\partial}{\partial x}\left(\frac{\partial L}{\partial U_{i, x}(t, x, b)}\right)\right\} \hat{U}_{i}(t, x, b) \\
+\frac{\partial}{\partial t \partial x}\left(\frac{\partial L}{\partial \dot{U}_{i, x}(t, x, b)}\right) \hat{U}_{i}(t, x, b) \\
+\frac{\partial}{\partial x^{2}}\left(\frac{\partial L}{\partial U_{i, x^{2}}(t, x, b)}\right) \hat{U}_{i}(t, x, b)
\end{array}\right] d t d x,
$$

$$
+\int_{t_{0}}^{t_{1}} \sum_{i=1}^{2}\left[\begin{array}{l}
\left.\frac{\partial L}{\partial U_{i, x}(t, x, b)} \hat{U}_{i}(t, x, b)\right|_{x_{0}} ^{x_{1}} \\
-\left.\frac{\partial}{\partial t}\left(\frac{\partial L}{\partial \dot{U}_{i, x}(t, x, b)}\right) \hat{U}_{i}(t, x, b)\right|_{x_{0}} ^{x_{1}} \\
+\left.\frac{\partial L}{\partial U_{i, x^{2}}(t, x, b)} \hat{U}(t, x, b)\right|_{x_{0}} ^{x_{1}} \\
-\left.\frac{\partial}{\partial x}\left(\frac{\partial L}{\partial U_{i, x^{2}}(t, x, b)} \hat{U}_{i}(t, x, b)\right)\right|_{x_{0}} ^{x_{1}}
\end{array}\right] d t=0 .
$$

Considering variations in $U_{i}$ as $U_{i}^{*}=U_{i}+\varepsilon \hat{U}_{i}$, one obtains: 
(3)

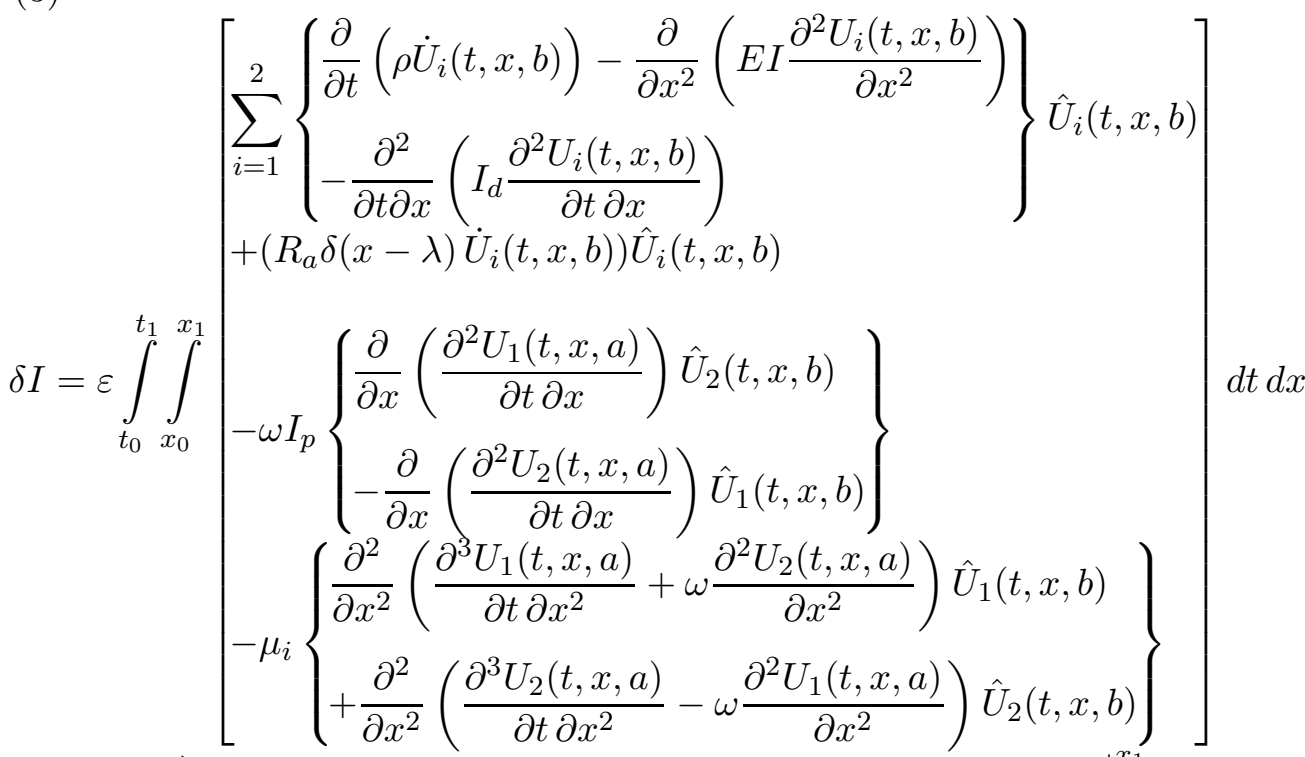

$$
\begin{aligned}
& +\left.\int_{t_{0}}^{t_{1}} \omega I_{p}\left\{\frac{\partial^{2} U_{1}(t, x, a)}{\partial t \partial x} \hat{U}_{2}(t, x, b)-\frac{\partial^{2} U_{2}(t, x, a)}{\partial t \partial x} \hat{U}_{1}(t, x, b)\right\}\right|_{x_{0}} ^{x_{1}} \\
& -\left.\left\{\begin{array}{l}
\sum_{i=1}^{2} \frac{\partial}{\partial x} E I \frac{\partial^{2} U_{i}(t, x, b)}{\partial x^{2}} \hat{U}_{i}(t, x, b) \\
+\frac{\partial}{\partial x} \mu_{i}\left\{\begin{array}{l}
\left(\frac{\partial^{3} U_{1}(t, x, a)}{\partial t \partial x^{2}}+\omega \frac{\partial^{2} U_{2}(t, x, a)}{\partial x^{2}}\right) \hat{U}_{1}(t, x, b)+ \\
\left(\frac{\partial^{3} U_{2}(t, x, a)}{\partial t \partial x^{2}}-\omega \frac{\partial^{2} U_{1}(t, x, a)}{\partial x^{2}}\right) \hat{U}_{2}(t, x, b)
\end{array}\right\} \\
-\frac{\partial}{\partial t} \sum_{i=1}^{2}\left(I_{d} \frac{\partial^{2} U_{i}(t, x, b)}{\partial t \partial x}\right) \hat{U}_{i}(t, x, b)
\end{array}\right\}\right|_{x_{0}} ^{t_{0}} \\
& +\sum_{i=1}^{2}\left\{E I \frac{\partial^{2} U_{i}(t, x, b)}{\partial x^{2}}\right\} \frac{\partial \hat{U}_{i}(t, x, b)}{\partial x} \\
& +\left.\mu_{i}\left\{\begin{array}{l}
\left(\frac{\partial^{3} U_{1}(t, x, a)}{\partial t \partial x^{2}}+\omega \frac{\partial^{2} U_{2}(t, x, a)}{\partial x^{2}}\right) \frac{\partial \hat{U}_{1}(t, x, b)}{\partial x} \\
+\left(\frac{\partial^{3} U_{2}(t, x, a)}{\partial t \partial x^{2}}-\omega \frac{\partial^{2} U_{1}(t, x, a)}{\partial x^{2}}\right) \frac{\partial \hat{U}_{2}(t, x, b)}{\partial x}
\end{array}\right\}\right|_{x_{0}} ^{x_{1}} d t=0
\end{aligned}
$$


All such notations are changed as $t \times a=t, t \times b=\eta$ with $U_{i}(t, x, b)=$ $u_{i}(\eta, x)$ and $U_{i}(t, x, a)=u_{i}(t, x)$, where $i=1,2$. After equating the coefficients of $U_{i}$ 's, the first part of the Eq. (3) provide umbra-field equations and the second part provide the boundary conditions at two ends of continuous shaft. The first part of the equation may be re-arranged in $\eta-t$ form, one may achieve umbra field after considering the limit $\eta \rightarrow t$ as:

(4a)

$$
\left[\begin{array}{l}
\left\{\begin{array}{l}
\frac{\partial}{\partial t}\left(\operatorname{Lim}_{\eta \rightarrow t} \rho \dot{u}_{1}(\eta, x)\right)+\frac{\partial}{\partial x^{2}}\left(\operatorname{Lim}_{\eta \rightarrow t} E I \frac{\partial^{2} u_{1}(\eta, x)}{\partial x^{2}}\right) \\
-\frac{\partial^{2}}{\partial t \partial x}\left(\operatorname{Lim}_{\eta \rightarrow t} I_{d} \frac{\partial^{2} u_{1}(\eta, x)}{\partial \eta \partial x}\right)+
\end{array}\right\} \\
+R_{a} \delta(x-\lambda) \frac{\partial}{\partial t}\left(\operatorname{Lim}_{\eta \rightarrow t} \dot{u}_{1}(\eta, x)\right) \\
-\omega I_{p}\left\{-\frac{\partial}{\partial x}\left(\frac{\partial^{2} u_{2}(t, x)}{\partial t \partial x}\right)\right\} \\
-\mu_{i}\left\{\frac{\partial^{2}}{\partial x^{2}}\left(\frac{\partial^{3} u_{1}(t, x)}{\partial t \partial x^{2}}+\omega \frac{\partial^{2} u_{2}(t, x)}{\partial x^{2}}\right)\right\}
\end{array}\right]=0
$$

and:

$$
\left[\begin{array}{l}
\left\{\begin{array}{l}
\frac{\partial}{\partial t}\left(\operatorname{Lim}_{\eta \rightarrow t} \rho \dot{u}_{2}(\eta, x)\right)+\frac{\partial}{\partial x^{2}}\left(\operatorname{Lim}_{\eta \rightarrow t} E I \frac{\partial^{2} u_{2}(\eta, x)}{\partial x^{2}}\right) \\
-\frac{\partial^{2}}{\partial t \partial x}\left(\operatorname{Lim}_{\eta \rightarrow t} I_{d} \frac{\partial^{2} u_{2}(\eta, x)}{\partial \eta \partial x}\right)
\end{array}\right\} \\
+R_{a} \delta(x-\lambda) \frac{\partial}{\partial t}\left(\operatorname{Lim}_{\eta \rightarrow t} \dot{u}_{2}(\eta, x)\right) \\
-\omega I_{p}\left\{\frac{\partial}{\partial x}\left(\frac{\partial^{2} u_{1}(t, x)}{\partial t \partial x}\right)\right\} \\
-\mu_{i}\left\{\frac{\partial^{2}}{\partial x^{2}}\left(\frac{\partial^{3} u_{2}(t, x)}{\partial t \partial x^{2}}-\omega \frac{\partial^{2} u_{1}(t, x)}{\partial x^{2}}\right)\right\}
\end{array}\right]=0
$$

The boundary conditions at each end, which may be expressed after 
taking limit $\eta \rightarrow t$ in Eq. (3), which may be represented as:

(5a)

$$
\left\{\begin{array}{l}
\omega I_{p}\left(-\frac{\partial^{2} u_{2}(t, x)}{\partial t \partial x}\right)-\frac{\partial}{\partial x}\left(\begin{array}{l}
E I \frac{\partial^{2} u_{1}(t, x)}{\partial x^{2}} \\
+\mu_{i}\left(\frac{\partial^{3} u_{1}(t, x)}{\partial t \partial x^{2}}+\omega \frac{\partial^{2} u_{2}(t, x)}{\partial x^{2}}\right)
\end{array}\right) \\
+\frac{\partial}{\partial t}\left(I_{d} \frac{\partial^{2} u_{1}(t, x)}{\partial t \partial x}\right)
\end{array}\right\}=0
$$

or $u_{1}(t, x)=0$.

$$
\left\{\begin{array}{l}
\omega I_{p}\left(-\frac{\partial^{2} u_{1}(t, x)}{\partial t \partial x}\right)-\frac{\partial}{\partial x}\left(\begin{array}{l}
E I \frac{\partial^{2} u_{2}(t, x)}{\partial x^{2}} \\
+\mu_{i}\left(\frac{\partial^{3} u_{2}(t, x)}{\partial t \partial x^{2}}+\omega \frac{\partial^{2} u_{1}(t, x)}{\partial x^{2}}\right)
\end{array}\right) \\
+\frac{\partial}{\partial t}\left(I_{d} \frac{\partial^{2} u_{2}(t, x)}{\partial t \partial x}\right)
\end{array}\right\}=0
$$

or $u_{2}(t, x)=0$.

$$
\begin{aligned}
& \left\{E I \frac{\partial^{2} u_{1}(t, x)}{\partial x^{2}}+\mu_{i}\left(\frac{\partial^{3} u_{1}(t, x)}{\partial t \partial x^{2}}+\omega \frac{\partial^{2} u_{2}(t, x)}{\partial x^{2}}\right)\right\}=0 \text { or } \\
& \frac{\partial u_{1}(t, x)}{\partial x}=0,
\end{aligned}
$$

$$
\begin{aligned}
& \left\{E I \frac{\partial^{2} u_{2}(t, x)}{\partial x^{2}}+\mu_{i}\left(\frac{\partial^{3} u_{2}(t, x)}{\partial t \partial x^{2}}+\omega \frac{\partial^{2} u_{1}(t, x)}{\partial x^{2}}\right)\right\}=0 \text { or } \\
& \frac{\partial u_{2}(t, x)}{\partial x}=0
\end{aligned}
$$

The different physical conditions at two ends may be reflected by the above boundary conditions. The first part in each of the above four pairs depicts shearing force balance and bending moment balance, and termed as dynamic boundary conditions. The second part in each pair provides displacement or slopes, and termed as geometric or essential boundary conditions.

\subsection{Classical Noether's theorem for fields}

Noether's theorem may be effectively employed to obtain an invariant of motion or conserved quantities for fields and continuous systems. The Noether's 
theorem [27] for fields states, that any differentiable symmetry of the action of a physical system has a corresponding conservation laws. It can also be defined, that for every differentiable symmetry generated by local actions, there corresponds a constant current. The action of a physical system is the integral over time of a Lagrangian function, from which the system behaviour can be formulated by the least action principle.

\subsection{Formulation of Extended Noether's theorem}

Formulation of Extended Noether's theorem may be obtained considering the methodology provided in Ref. [9] and briefly presented in Appendix for ready reference of the readers. Some basic concepts of umbra-Lagrangian density have been provided in reference $[6,7,11]$.

The prolonged infinitesimal generators [32, 33 and 34] are composed of two parts as

$$
p_{r}^{(3)} V=p_{r}^{(3)} V_{\eta}+p_{r}^{(3)} V_{t}
$$

where, $p_{r}^{(3)} V_{\eta}$ and $p_{r}^{(3)} V_{t}$ are the prolonged umbra-time and real-time component the infinitesimal generators $p_{r}^{(3)} V$. One may achieve the invariance of umbraLagrangian density, which may now be expressed as:

$$
p_{r}^{(3)} V(L)=0 .
$$

One may formulate the Extended Noether's theorem through substitution and taking the limit $\eta \rightarrow t$,

$$
\operatorname{Lim}_{\eta \rightarrow t}\left\{p_{r}^{(3)} V_{\eta}(L)\right\}+\operatorname{Lim}_{\eta \rightarrow t}\left\{p_{r}^{(3)} V_{t}(L)\right\}=0
$$

The first term of the Eq. (4a) may be represented as:

(9) $\operatorname{Lim}_{\eta \rightarrow t}(t, x) p_{r}^{(3)(t, x)} V_{\eta}(L)$

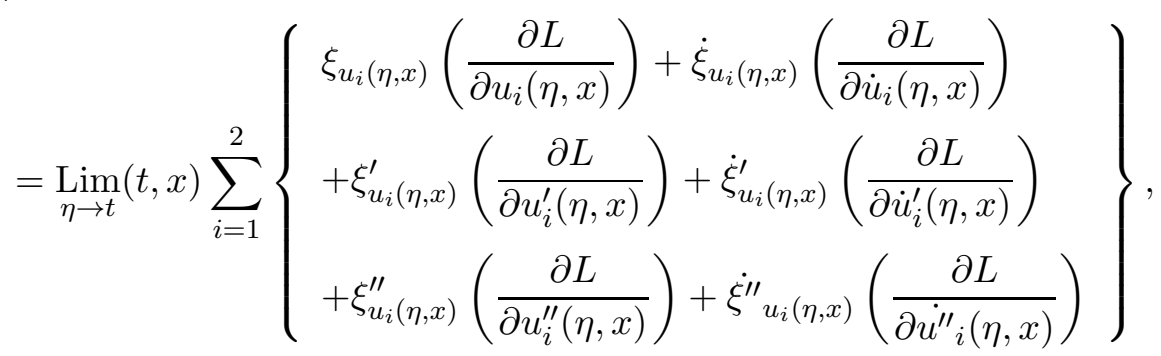


All above notions in details are provided in Appendix. Expanding each term with derivatives of $\xi$ 's in Eq. (9) yields after simplification as:

$$
\left.\begin{array}{l}
\text { (10) } \operatorname{Lim}_{\eta \rightarrow t} p_{r}^{(3)} V_{\eta}(L) \\
=\operatorname{Lim}_{\eta \rightarrow t} \sum_{i=1}^{2}\left\{\begin{array}{l}
\xi_{u_{i}(\eta, x)}\left(\frac{\partial L}{\partial \dot{u}_{i}(\eta, x)}\right)+\frac{\partial}{\partial x}\left(\xi_{u_{i}(\eta, x)}\left(\frac{\partial L}{\partial \dot{u}_{i}^{\prime}(\eta, x)}\right)\right) \\
-\xi_{u_{i}(\eta, x)} \frac{\partial}{\partial x}\left(\frac{\partial L}{\partial \dot{u}_{i}^{\prime}(\eta, x)}\right)
\end{array}\right\} \\
+D_{x}\left\{\begin{array}{l}
\xi_{u_{i}(\eta, x)}\left(\frac{\partial L}{\partial u_{i}^{\prime}(\eta, x)}\right)-\left(\xi_{u_{i}(\eta, x)} \frac{\partial}{\partial t}\left(\frac{\partial L}{\partial \dot{u}_{i}^{\prime}(\eta, x)}\right)\right) \\
+\xi_{u_{i}(\eta, x)}^{\prime}\left(\frac{\partial L}{\partial u_{i}^{\prime \prime}(\eta, x)}\right)-\xi_{u_{i}(\eta, x)} \frac{\partial}{\partial x}\left(\frac{\partial L}{\partial u_{i}^{\prime \prime}(\eta, x)}\right)
\end{array}\right\}
\end{array}\right],
$$

where $D_{t}$ is the total time derivative and $D_{x}$ is the total space derivatives, respectively and may be mathematically explained as:

$$
\begin{gathered}
D_{t} Z_{1}\left(t, x, u, u_{x}\right)=\frac{\partial Z_{1}}{\partial t}+\frac{\partial Z_{1}}{\partial u} \frac{\partial u}{\partial t}+\frac{\partial Z_{2}}{\partial u_{x}} \frac{\partial u_{x}}{\partial t} \\
D_{x} Z_{2}\left(t, x, u, u_{x}, u_{x^{2}}\right)=\frac{\partial Z_{2}}{\partial x}+\frac{\partial Z_{2}}{\partial u} \frac{\partial u}{\partial x}+\frac{\partial Z_{2}}{\partial u_{x}} \frac{\partial u_{x}}{\partial x}+\frac{\partial Z_{2}}{\partial u_{x^{2}}} \frac{\partial u_{x^{2}}}{\partial x},
\end{gathered}
$$

and functions $Z_{1}$ and $Z_{2}$ may explained as:

$$
\begin{array}{r}
Z_{1}=\operatorname{Lim}_{\eta \rightarrow t} \sum_{i=1}^{2}\left\{\xi_{u_{i}(\eta, x)}\left(\frac{\partial L}{\partial \dot{u}_{i}(\eta, x)}\right)+\frac{\partial}{\partial x}\left(\xi_{u_{i}(\eta, x)}\left(\frac{\partial L}{\partial \dot{u}_{i}^{\prime}(\eta, x)}\right)\right)\right. \\
\left.-\xi_{u_{i}(\eta, x)} \frac{\partial}{\partial x}\left(\frac{\partial L}{\partial \dot{u}_{i}^{\prime}(\eta, x)}\right)\right\}, \\
Z_{2}=\operatorname{Lim}_{\eta \rightarrow t} \sum_{i=1}^{2}\left\{\begin{array}{c}
\xi_{u_{i}(\eta, x)}\left(\frac{\partial L}{\partial u_{i}^{\prime}(\eta, x)}\right)-\xi_{u_{i}(\eta, x)} \frac{\partial}{\partial t}\left(\frac{\partial L}{\partial \dot{u}_{i}^{\prime}(\eta, x)}\right) \\
+\xi_{u_{i}(\eta, x)}^{\prime}\left(\frac{\partial L}{\partial u_{i}^{\prime \prime}(\eta, x)}\right)-\xi_{u_{i}(\eta, x)} \frac{\partial}{\partial x}\left(\frac{\partial L}{\partial u_{i}^{\prime \prime}(\eta, x)}\right)
\end{array}\right\} .
\end{array}
$$

Thus, one may re-write Eq. (10) as:

$$
\operatorname{Lim}_{\eta \rightarrow t} p_{r}^{(3)} V_{\eta}(L)=D_{t} Z_{1}+D_{x} Z_{2} .
$$


After Substitution of Eq. (15) in Eq. (8) finally results in extended Noether's theorem for fields, which may be expressed as:

$$
D_{t} Z_{1}+D_{x} Z_{2}+\lim _{\eta \rightarrow t} P_{r}^{3} V_{t}(L)=0 .
$$

Where functions $Z_{1}$ and $Z_{2}$ may be defined as:

$$
\begin{aligned}
& Z_{1}=\lim _{\eta \rightarrow t}\left[\sum_{i=1}^{2} \xi_{i}(\eta)\left\{\begin{array}{c}
\frac{\partial L}{\partial \dot{u}_{i}(\eta, x)}-\frac{d}{d x}\left(\frac{\partial L}{\partial \dot{u}_{i x}(\eta, x)}\right) \\
+\frac{d^{2}}{d x^{2}}\left(\frac{\partial L}{\partial \dot{u}_{i x x}(\eta, x)}\right)
\end{array}\right\}\right], \\
& Z_{2}=\lim _{\eta \rightarrow t}\left[\sum_{i=1}^{2}\left(\begin{array}{l}
\xi_{i}(\eta) \frac{\partial L}{\partial u_{i x}(\eta, x)}+\xi_{i}^{\prime}(\eta)\left(\frac{\partial L}{\partial u_{i x x}(\eta, x)}\right) \\
-\xi_{i}(\eta) \frac{d}{d x}\left(\frac{\partial L}{\partial u_{i x x}(\eta, x)}\right)
\end{array}\right)\right] \\
& +\lim _{\eta \rightarrow t}\left[\sum_{i=1}^{2}\left(\begin{array}{l}
\dot{\xi}_{i}(\eta) \frac{\partial L}{\partial \dot{u}_{i x}(\eta, x)}+\dot{\xi}_{i}^{\prime}(\eta)\left(\frac{\partial L}{\partial \dot{u}_{i x x}(\eta, x)}\right) \\
-\dot{\xi}_{i}(\eta) \frac{d}{d x}\left(\frac{\partial L}{\partial \dot{u}_{i x x}(\eta, x)}\right)
\end{array}\right) .\right.
\end{aligned}
$$

Equation (16) is represented as an extended Noether's field equation for the umbra-Lagrangian density, where $Z_{1}$ may be defined as local density, $Z_{2}$ as current or flux density (It is more often defined as Noether's current density) and the last additional term may be defined as the modulatory convection term, which is due to the contribution of nonconservative actions and gyroscopic currents, including the dissipation effects, which may also be assumed as local rate of production.

\section{Formulation of continuous rotor shaft with in-span external}

\section{damping}

In this case study, a rotor shaft with in-span concentrated discrete damper has been considered in the system. The rotor shaft material comprises of internal damping and shaft is driven by a constant supply motor. The motor is attached with continuation shaft with an aid of dissipated coupling, as shown in Fig. 2. 


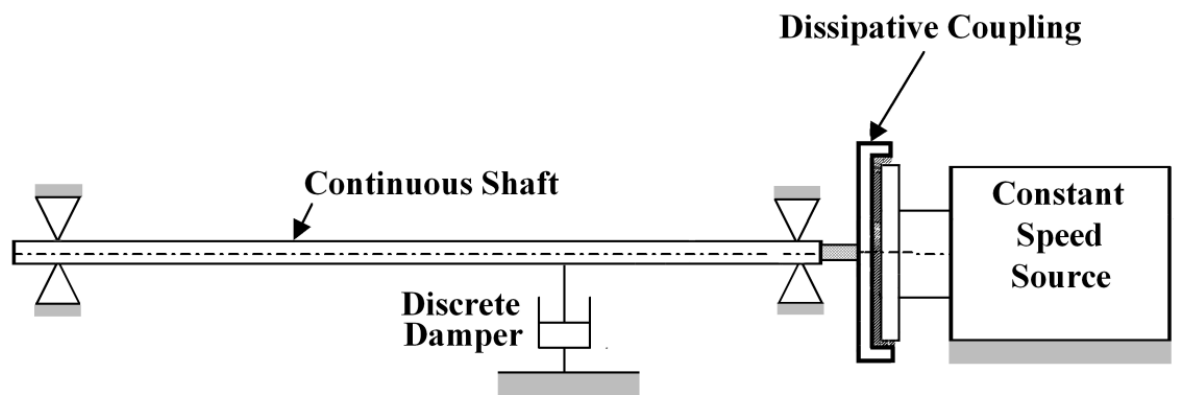

Fig. 2. Continuous rotor shaft with in-span concentrated discrete damper

One may obtain umbra-Lagrangian Density of the system, which may be written as:

$$
\begin{aligned}
& L=\int_{x_{0}}^{x_{1}}\left[\begin{array}{c}
\sum_{i=1}^{2}\left\{\begin{array}{c}
\frac{1}{2} \rho\left(\frac{\partial u_{i}(\eta, x)}{\partial \eta}\right)^{2}-\frac{1}{2} E I\left(\frac{\partial^{2} u_{i}(\eta, x)}{\partial x^{2}}\right)^{2} \\
-\frac{1}{2} I_{d}\left(\frac{\partial^{2} u_{i}(\eta, x)}{\partial \eta \partial x}\right)^{2}
\end{array}\right\} \\
-\mu_{i}\left\{\begin{array}{c}
\left(\frac{\partial^{3} u_{1}(t, x)}{\partial t \partial x^{2}}+\dot{\theta}(t) \frac{\partial^{2} u_{2}(t, x)}{\partial x^{2}}\right) \frac{\partial^{2} u_{1}(\eta, x)}{\partial x^{2}} \\
+\left(\frac{\partial^{3} u_{2}(t, x)}{\partial t \partial x^{2}}-\dot{\theta}(t) \frac{\partial^{2} u_{1}(t, x)}{\partial x^{2}}\right) \frac{\partial^{2} u_{2}(\eta, x)}{\partial x^{2}}
\end{array}\right\}
\end{array}\right] d x \\
& \int_{x_{0}}^{x_{1}} R_{a} \delta(x-\lambda)\left(u_{1} \dot{u}_{2}-u_{2} \dot{u}_{1}\right) d x \\
& -\left[\begin{array}{l}
\mu_{i} \int_{x_{0}}^{x_{1}}\left\{\frac{\partial^{2} u_{1}(t, x)}{\partial x^{2}} \frac{\partial^{3} u_{2}(t, x)}{\partial t \partial x^{2}}-\frac{\partial^{2} u_{2}(t, x)}{\partial x^{2}} \frac{\partial^{3} u_{1}(t, x)}{\partial t \partial x^{2}}\right\} d x \\
+R_{c}(\dot{\theta}(t)-\Omega)
\end{array}\right] \theta(\eta) \\
& +\frac{1}{2} J \dot{\theta}^{2}(\eta),
\end{aligned}
$$

where $R_{c}$ is damping coefficient of a dissipative coupling, $R_{a}$ is the damping coefficient of in-span external damper, and $\Omega$ is the excitation frequency. In Eq. (19), the first term is kinetic energy of the system, the second term is strain energy for small deflections, where $E$ is the Young's modulus of the shaft material, and $I$ is the cross-section moment of inertia, the third term is 
contributed by rotary moments with the angular velocity $\frac{\partial^{2} u_{i}(\eta, x)}{\partial \eta \partial x}$, the fourth term is contributed by gyroscopic forces with constant angular velocity $\omega$, the fifth term is contributed by discrete external damper, and the sixth term is umbra-potential, due to internal damping. All terms are in per unit length, In Eq. (19), the fifth term contributed by external damping is very significant. For this system with in-span discrete external damping, the term, which needs special consideration, is:

$$
\int_{0}^{L} R_{a} \delta(x-\lambda)\left(u_{1} \dot{u}_{2}-u_{2} \dot{u}_{1}\right) d x, \text { where } \lambda \in(0, L) .
$$

In this case, $\delta$ is Dirac delta function. It is assumed the following form of motion as:

$$
u_{1}=\sum_{n=1}^{\infty} A_{n} \cos \Omega_{n} t \sin \frac{n \pi}{L} x \text { and, } u_{2}=\sum_{n=1}^{\infty} A_{n} \sin \Omega_{n} t \sin \frac{n \pi}{L} x
$$

where $A_{n}$ may be defined as slowly varying function with time.

Substitution of Eq. (21) into Eq. (20) yields:

$$
R_{a}\left(\Omega_{n} \sum_{n=1}^{\infty} \sum_{m=1}^{\infty} A_{n} A_{m} \sin \frac{n \pi}{L} \lambda \sin \frac{m \pi}{L} \lambda\right)
$$

The above expression may be used in extended Noether's theorem to evaluate the amplitude equation of the system.

3.1 Formulation of extended Noether's theorem for shaft with in-span concentrated discrete damper

The extended Noether's rate with in-span concentrated damping may be written as:

$$
\left[\begin{array}{l}
\sum_{n=1}^{\infty} \frac{L}{2} \Omega_{n} A_{n}\left\{\dot{A}_{n}+\frac{1}{2 \rho}\left(\mu_{i} \frac{n^{4} \pi^{4}}{L^{4}}\right) A_{n}\right\}-\frac{\mu_{i} n^{4} \pi^{4}}{2 \rho L^{4}} \dot{\theta}(t) A_{n}^{2} \\
+\frac{\Omega_{n} R_{a}}{2 \rho} \sum_{n=1}^{\infty} \sum_{m=1}^{\infty} A_{n} A_{m} \sin \frac{n \pi}{L} \lambda \sin \frac{m \pi}{L} \lambda
\end{array}\right]=0
$$




$$
\begin{aligned}
\sum_{n=1}^{\infty} A_{n}\left[\dot{A}_{n}+\frac{1}{2 \rho}\left\{\mu_{i} \frac{n^{4} \pi^{4}}{L^{4}}-\right.\right. & \left.\left.\frac{\mu_{i} n^{4} \pi^{4}}{\Omega_{n} L^{4}} \dot{\theta}(t)\right\} A_{n}\right] \times \frac{L}{2} \\
& +\frac{R_{a}}{2 \rho} \sum_{j=1}^{\infty} \sum_{k=1}^{\infty} A_{j} A_{k} \sin \frac{j \pi}{L} \lambda \sin \frac{k \pi}{L} \lambda=0,
\end{aligned}
$$

or it is written as:

$$
\begin{aligned}
\frac{L}{2} \sum_{n=1}^{\infty} A_{n}\left[\dot{A}_{n}+\frac{1}{2 \rho}\left\{\mu_{i} \frac{n^{4} \pi^{4}}{L^{4}}-\frac{\mu_{i} n^{4} \pi^{4}}{\Omega_{n} L^{4}} \dot{\theta}(t)\right\} A_{n}\right. & \\
& \left.+\frac{R_{a}}{\rho L} \sum_{k=1}^{\infty} A_{k} \sin \frac{n \pi}{L} \lambda \sin \frac{k \pi}{L} \lambda\right]=0 .
\end{aligned}
$$

One may consider independent variations in $A_{n}+\delta A_{n}$ and after dropping the factor $L / 2$, the variational equation may be expressed as:

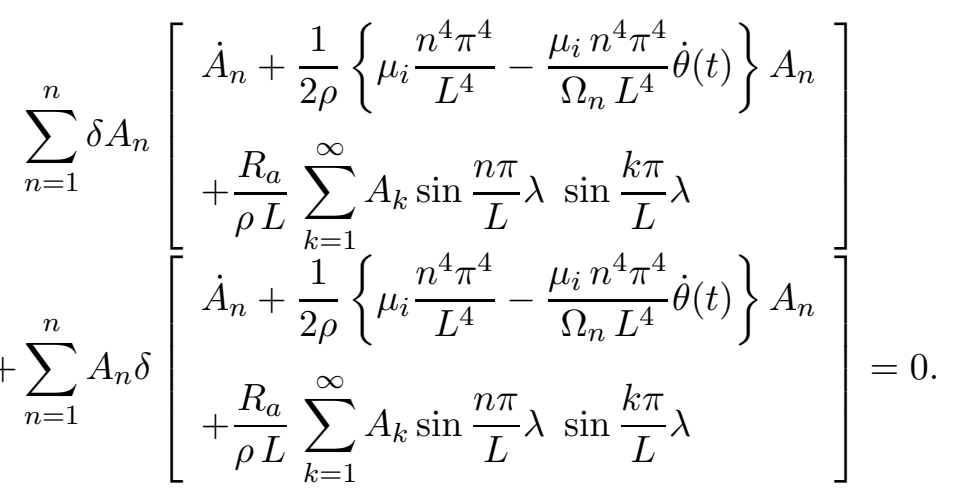

One may deduce the symmetry condition, which will be valid for neighbourhood paths. The following conditions are essentially required to be satisfied, given as:

$$
\begin{aligned}
& \dot{A}_{n}+\frac{1}{2 \rho}\left\{\mu_{i} \frac{n^{4} \pi^{4}}{L^{4}}-\frac{\mu_{i} n^{4} \pi^{4}}{\Omega_{n} L^{4}} \dot{\theta}(t)\right\} A_{n} \\
& +\frac{R_{a}}{\rho L} \sum_{k=1}^{\infty} A_{k} \sin \frac{n \pi}{L} \lambda \sin \frac{k \pi}{L} \lambda=0 .
\end{aligned}
$$

One may examine the condition for entrainment of the $n^{\text {th }}$ mode if $A_{n} \rightarrow$ finite limit and $A_{k} \rightarrow 0$. This is possible only if $k \neq n$ for $t \rightarrow \infty$, which 
finally resulted into:

$$
\frac{1}{2 \rho}\left\{\mu_{i} \frac{n^{4} \pi^{4}}{L^{4}}-\frac{\mu_{i} n^{4} \pi^{4}}{\Omega_{n} L^{4}} \dot{\theta}(t)\right\}+\frac{R_{a}}{\rho L} \sin ^{2} \frac{n \pi}{L} \lambda=0 .
$$

The value of $\dot{\theta}(t)$ will be obtained from Eq. (28) and written as:

$$
\frac{1}{2 \rho} \frac{\mu_{i} n^{4} \pi^{4}}{\Omega_{n} L^{4}} \dot{\theta}(t)=\frac{1}{2 \rho} \mu_{i} \frac{n^{4} \pi^{4}}{L^{4}}+\frac{R_{a} \times 2}{\rho L \times 2} \sin ^{2} \frac{n \pi}{L} \lambda,
$$

or

$$
\dot{\theta}(t)=\Omega_{n}\left[1+\frac{2 R_{a} L^{3}}{\mu_{i} n^{4} \pi^{4}} \sin ^{2} \frac{n \pi}{L} \lambda\right] .
$$

\subsection{Umbra-Hamiltonian density of continuous rotor system}

It is required to evaluate umbra-Hamiltonian density of the system to achieve the amplitude equation of the shaft, to achieve an amplitude equation of the continuous rotor system with in-span damper. Umbra-Hamiltonian [9] may be expressed as:

$$
\begin{aligned}
& H=\int_{x_{0}}^{x_{1}}\left[\begin{array}{c}
\sum_{i=1}^{2}\left\{\begin{array}{c}
\frac{1}{2 \rho} P_{i}^{2}(\eta, x)+\frac{1}{2} E I\left(\frac{\partial^{2} u_{i}(\eta, x)}{\partial x^{2}}\right)^{2} \\
+\frac{1}{2} I_{d}\left(\frac{\partial^{2} u_{i}(\eta, x)}{\partial \eta \partial x}\right)^{2}
\end{array}\right\} \\
+\mu_{i}\left\{\begin{array}{c}
\left(\frac{\partial^{3} u_{1}(t, x)}{\partial t \partial x}+\dot{\theta}(t) \frac{\partial^{2} u_{2}(t, x)}{\partial x^{2}}\right) \frac{\partial^{2} u_{1}(\eta, x)}{\partial x^{2}} \\
+\left(\frac{\partial^{3} u_{2}(t, x)}{\partial t \partial x}-\dot{\theta}(t) \frac{\partial^{2} u_{1}(t, x)}{\partial x^{2}}\right) \frac{\partial^{2} u_{2}(\eta, x)}{\partial x^{2}}
\end{array}\right\}
\end{array}\right] d x \\
& +\int_{x_{0}}^{x_{1}} R_{a}(x-\lambda)\left(u_{1} \dot{u}_{2}-u_{2} \dot{u}_{1}\right) d x \\
& +\left[\begin{array}{l}
\mu_{i} \int_{x_{0}}^{x_{1}}\left\{\frac{\partial^{2} u_{1}(t, x)}{\partial x^{2}} \frac{\partial^{3} u_{2}(t, x)}{\partial t \partial x^{2}}-\frac{\partial^{2} u_{2}(t, x)}{\partial x^{2}} \frac{\partial^{3} u_{1}(t, x)}{\partial t \partial x^{2}}\right\} d x \\
+R_{c}(\dot{\theta}(t)-\Omega)
\end{array}\right] \theta(\eta) \\
& +\frac{1}{2 J_{d}} P_{\theta}^{2} \text {, }
\end{aligned}
$$


where $P_{i}(\eta, x)=\rho \frac{\partial u_{i}(\eta, x)}{\partial \eta}$ and $P_{\theta}(\eta)=J_{d} \dot{\theta}(\eta)$.

As discussed in reference [9], one may have the similar theorems for umbra-Hamiltonian density, which may be expressed as:

$$
\operatorname{Lim}_{\eta \rightarrow t} \frac{\partial H^{*}}{\partial \eta}=0 \Rightarrow \frac{d H_{i}^{*}}{d t}=-\operatorname{Lim}_{\eta \rightarrow t} \frac{d H_{e}^{*}}{\partial \eta} .
$$

Considering the equation (2), one may evaluate exterior umbra-Hamiltonian density, expressed as:

$$
\begin{aligned}
\operatorname{Lim}_{\eta \rightarrow t} \frac{\partial H_{e}}{\partial \eta}=\int_{x_{0}}^{x_{1}}\left[\begin{array}{l}
R_{a}(x-\lambda)\left(u_{1} \dot{u}_{2}-u_{2} \dot{u}_{1}\right) d x \\
+\mu_{i}\left[\begin{array}{l}
\left\{\frac{\partial^{3} u_{1}(t, x)}{\partial t \partial x^{2}}+\dot{\theta}(t) \frac{\partial^{2} u_{2}(t, x)}{\partial x^{2}}\right\} \frac{\partial^{3} u_{1}(t, x)}{\partial t \partial x^{2}} \\
+\left\{\frac{\partial^{3} u_{2}(t, x)}{\partial t \partial x^{2}}-\dot{\theta}(t) \frac{\partial^{2} u_{1}(t, x)}{\partial x^{2}}\right\} \frac{\partial^{3} u_{2}(t, x)}{\partial t \partial x^{2}}
\end{array}\right] d x
\end{array}\right] \\
+\left[\begin{array}{l}
\mu_{i} \int_{x_{0}}^{x_{1}}\left\{\begin{array}{l}
\frac{\partial^{2} u_{1}(t, x)}{\partial x^{2}} \frac{\partial^{3} u_{2}(t, x)}{\partial t \partial x^{2}} \\
+R_{c}\left(\dot{\theta}(t)-\frac{\partial^{2} u_{2}(t, x)}{\partial x^{2}} \frac{\partial^{3} u_{1}(t, x)}{\partial t \partial x^{2}}\right.
\end{array}\right\} d x \\
\dot{\theta}(t)=0 .
\end{array}\right]
\end{aligned}
$$

The end conditions of the continuous shaft have been taken as pin-pin considering self-aligning bearing. After substituting Eq. (21) in Eq. (33), one obtains the following two terms distinguished, as $\{P\}$ and $\{Q\}$ :

$$
\begin{aligned}
& \operatorname{Lim}_{\eta \rightarrow t} \frac{\partial H_{e}}{\partial \eta}=R_{a} \Omega_{n} \sum_{n=1}^{\infty} \sum_{m=1}^{\infty} A_{n} A_{m} \sin \frac{n \pi}{L} x \sin \frac{m \pi}{L} x \\
& +\mu_{i} \frac{L}{2}\left[\begin{array}{l}
\sum_{n=1}^{\infty} A_{n}^{2} \Omega_{n}^{2}\left(\frac{n \pi}{L}\right)^{4} \\
\left.+\dot{\theta}(t) \sum_{n=1}^{\infty} A_{n}^{2} \Omega_{n}\left(\frac{n \pi}{L}\right)^{4}+\sum_{n=1}^{\infty} \dot{A}_{n}^{2}\left(\frac{n \pi}{L}\right)^{4}\right]
\end{array}\right] \\
& +\left[\mu_{i}\left\{\int_{0}^{L} \sum_{n=1}^{\infty} A_{n}^{2} \Omega_{n} \sin ^{2} \frac{n \pi x}{L}\left(\frac{n \pi}{L}\right)^{4}\right\} d x+R_{c}(\dot{\theta}(t)-\Omega)\right] \dot{\theta}(t) .
\end{aligned}
$$


Equating term $\{P\}$ to zero, one obtains the expression:

$$
\dot{A}_{n}^{2}+A_{n}^{2} \Omega_{n}\left[\frac{\mu_{i} \Omega_{n} n^{4} \pi^{4}}{L^{4}}-\frac{\mu_{i} n^{4} \pi^{4}}{L^{4} \Omega_{n}} \dot{\theta}(t)+\frac{2 R_{a}}{L} \sin ^{2} \frac{n \pi}{L} \lambda\right]=0 .
$$

Considering independent variations in $A_{n}+\delta A_{n}$, the variation equation may be written similar to Eq. (26), after dropping the factor $L / 2$. Repeating the same steps from Eq. (27) to (28), one obtains the value of $\dot{\theta}(t)$ as:

$$
\dot{\theta}(t)=\Omega_{n}\left[1+\frac{2 R_{a} L^{3}}{\mu_{i} n^{4} \pi^{4}} \sin ^{2} \frac{n \pi}{L} \lambda\right] .
$$

The Eq. (35) makes the term $\{P\}$ zero and also resembles with Eq. (30). After equating the second term $\{Q\}$ to zero, one may obtain:

$$
\mu_{i} \sum_{n=1}^{\infty} A_{n}^{2} \Omega_{n}\left(\frac{n \pi}{L}\right)^{4} \int_{0}^{L} \phi_{n}^{2} d x+R_{c}(\dot{\theta}(t)-\Omega)=0 .
$$

Considering the shaft's speed is entrained at

$$
\dot{\theta}(t)=\Omega_{n}\left[1+\frac{2 R_{a} L^{3}}{\mu_{i} n^{4} \pi^{4}} \sin ^{2} \frac{n \pi}{L} \lambda\right],
$$

then all the amplitudes converge to zero except $A_{n}$, thus, the amplitude equation of the shaft with in- span damping may be obtained as:

$$
A_{n}=\sqrt{\frac{2 R_{c}\left(\Omega-\operatorname{Min}_{n \in I^{+}} \Omega_{n}\left[1+\frac{2 R_{a} L^{3}}{\mu_{i} n^{4} \pi^{4}} \sin ^{2} \frac{n \pi}{L} \lambda\right]\right)}{\mu_{i} \frac{n^{4} \pi^{4}}{L^{3}} \Omega_{n}}},
$$

or

$$
A_{n}=\sqrt{\frac{2 R_{c}\left(\Omega-\operatorname{Min}_{n \in I^{+}} \Omega_{n}\left[1+\frac{2 R_{a} L^{3}}{\mu_{i} n^{4} \pi^{4}} \sin ^{2} \frac{n \pi}{L} \lambda\right]\right)}{\zeta_{n} \Omega_{n}}},
$$

where $\zeta_{n}=\mu_{i} \frac{n^{4} \pi^{4}}{L^{3}}$. The next section presents the modelling simulation of rotating shaft with in span concentrated damper. 
4. Modelling and simulation of rotating shaft with in- span discrete external damping

The physical system of discrete-continuous rotor shaft with in-span discrete external damper has been shown in Fig. 2. The shaft is driven by a constant supply voltage source through dissipative coupling. Any DC motor may qualify as a constant supply voltage source. The self aligning bearing is employed and the coupling of the system is assumed to be flexible in transverse and longitudinal direction, but at the same time, it is torsion rigid, as torsion vibrations are not prominent in comparison to transverse and flexural vibrations. The bond graphs are conveniently used as a modelling technique as it portrays the system from its physical paradigms. The object oriented reusable capsules, called vector bond graphs of the system, shown in Fig. 3 are created with in-span discrete external damping.

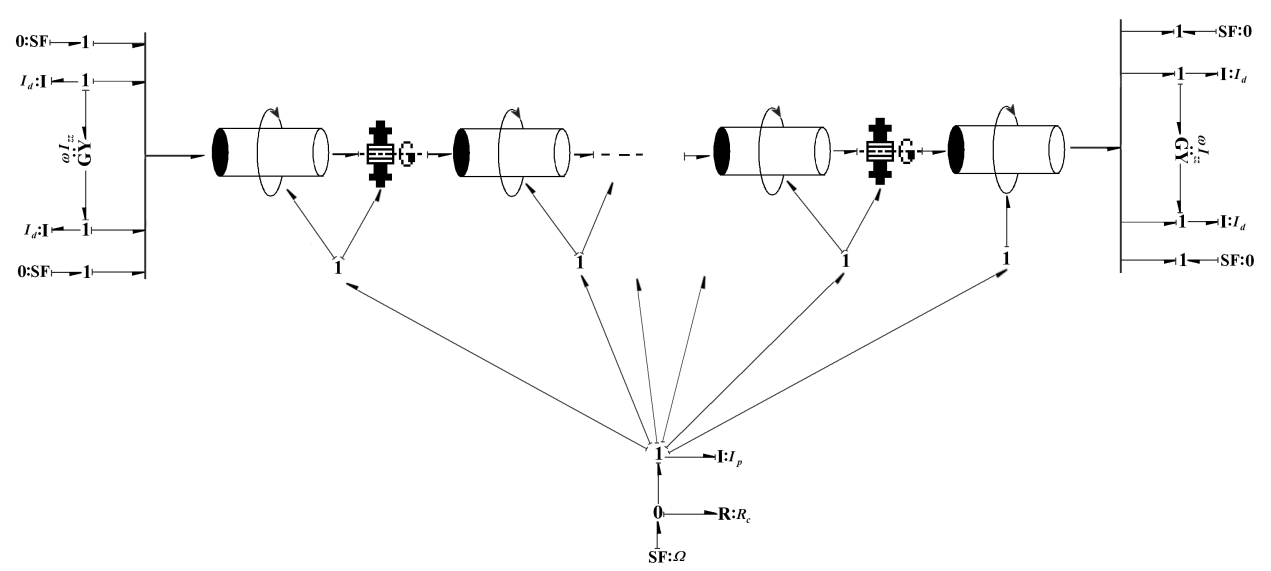

Fig. 3. Bond graph model of integrated system of rotor shaft with in-span concentrated external damper

\subsection{Simulation studies}

The bond graph model $[11,28,29]$ of the continuous shaft with in span concentrated external damping is simulated in SYMBOLS-Sonata [30, 31] software, in order to visualize the complex modes of the system. However, the external damping in sub models of shaft has been considered as discrete or finite. In this case, the effects of concentrated damper are closely linked to the complex character of modes. The simulation rig consists of a hollow rotating shaft with hub elements. In this simulation 10 reticules are used, so that flexural behaviour may be apparent. The two ends are well supported on 
a self-aligning bearing, so as adjust any kind of misalignment. The length of the beam is taken as $5 \mathrm{~m}$ long and external and internal diameters are $0.02 \mathrm{~m}$ and $0.01 \mathrm{~m}$, respectively. The elasticity of material is taken as $104.5 e^{9} \mathrm{~N} / \mathrm{m}^{2}$ and density of material is taken as $4420 \mathrm{~kg} / \mathrm{m}^{3}$. The damping coefficient of dissipative coupling is considered as $R_{c}=0.002 \mathrm{Ns} / \mathrm{m}$. It has been assumed, that in-span discrete damper permits motion to the rotor and at the same time leads to substantial energy dissipation.

Simulation is performed for the parameters, as given in Table-1, considering discrete external damping, $R_{a}=0.020$ and excitation frequency, $\Omega=5$ $\mathrm{Hz}$ or $31.41 \mathrm{rad} / \mathrm{s}$. To reduce the simulation time and running the simulation effectively, an initial momentum of $0.001 \mathrm{~kg}-\mathrm{m}^{2}$ was also given to $3^{\text {rd }}$ reticule. Figure 4 shows the limit orbits of the rotor shaft, due to loading of the motor. The significant feature of the simulation is that the spinning speed of the shaft gets latched at $22.392 \mathrm{rad} / \mathrm{s}$, as the first critical speed of instability, $\dot{\theta}_{1^{\text {st }}} \bmod e$ and matches nearly with the calculated value of shaft spinning speed (latched) $\dot{\theta}(t)$ (Table 1), which is equal to $22.266 \mathrm{rad} / \mathrm{s}$. The analysis has been done at the point of contact with the discrete external damping.

Table 1. Simulation parameters

\begin{tabular}{|c|c|}
\hline Parameter & Value \\
\hline Length of rotor & $L_{\text {beam }}=5 \mathrm{~m}$ \\
\hline Number of elements & $N_{\text {elem }}=10$ \\
\hline $\begin{array}{l}\text { Modulus of elasticity for the rotor shaft } \\
\text { material }\end{array}$ & $E=104.5 e 9 \mathrm{~N} / \mathrm{m}^{2}$ \\
\hline Density of the rotor shaft material & $\rho=4420 \mathrm{~kg} / \mathrm{m}^{3}$ \\
\hline Internal radius of the shaft & $R_{i}=0.01 \mathrm{~m}$ \\
\hline External radius of the shaft & $\mathrm{R}_{o}=0.02 \mathrm{~m}$ \\
\hline Dissipative coupling coefficient & $R_{c}=0.002$ N.m.s \\
\hline Excitation frequency & $\begin{array}{l}\Omega=5 \mathrm{H} \mathrm{z} \text { (for first mode) } \\
\Omega=30 \mathrm{~Hz} \text { (for second mode) }\end{array}$ \\
\hline $\begin{array}{l}\text { Internal damping coefficient of the rotor } \\
\text { shaft }\end{array}$ & $\mu_{i}=1.0 e-4 \mathrm{Ns} / \mathrm{m}$ \\
\hline $\begin{array}{l}\text { Discrete External Damping Coefficient } \\
\text { of the rotor shaft }\end{array}$ & $\begin{array}{l}R_{a}=0.020 \mathrm{Ns} / \mathrm{m} \text { (for first mode) } \\
R_{a}=6.00 \mathrm{Ns} / \mathrm{m} \text { (for second mode) }\end{array}$ \\
\hline $\begin{array}{l}\text { Shaft spinning speed (entrained) calcu- } \\
\text { lated }\end{array}$ & $\begin{array}{l}\dot{\theta}(t)=22.392 \mathrm{rad} / \mathrm{s}, 95.405 \mathrm{rad} / \mathrm{s} \\
(\text { For Mode } 1,2, \text { respectively) }\end{array}$ \\
\hline
\end{tabular}

Further, coefficient of damping for in-span concentrated discrete damper is increased to $6.0 \mathrm{Ns} / \mathrm{m}$ and excitation frequency $\Omega$ of the motor is increased to $30 \mathrm{~Hz}$ or $188.327 \mathrm{rad} / \mathrm{sec}$. One may achieve the second threshold speed of 


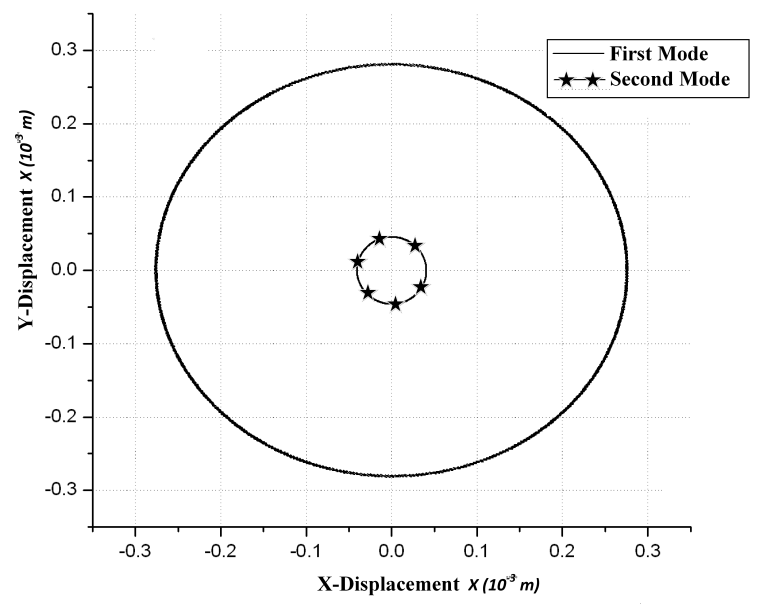

Fig. 4. Limiting orbit of rotating shaft (at point of contact with in span discrete external damping) at first two undamped natural modes

Table 2. Comparison of natural frequency

\begin{tabular}{|l|c|c|c|}
\hline Description & $\begin{array}{c}\text { Calculated } \\
\text { Value }\end{array}$ & $\begin{array}{c}\text { Numerical } \\
\text { Value }\end{array}$ & $\%$ Error \\
\hline First mode natural frequency & $3.414 \mathrm{~Hz}$ & $3.400 \mathrm{~Hz}$ & $0.41 \%$ \\
\hline Second mode natural frequency & $13.66 \mathrm{~Hz}$ & $13.600 \mathrm{~Hz}$ & $0.44 \%$ \\
\hline
\end{tabular}

the shaft, shown in Fig. 3, as second mode. It is depicted from the figure, that the amplitudes of first two natural modes as trajectories of limit orbits, which are superimposed on the same plot. The simulated value of the natural modes machetes nearly exact to the calculated natural modes, as given in Table 2 . The interesting phenomenon, observed from this simulation, is that one obtains the natural undamped modes in this case. The result may also be analysed through Fast Fourier Transform (FFT) analyser tool, as shown in Figs 5(a)-5(b), where first two natural frequencies for natural modes are superimposed.

The animation of the limiting orbits is also carried out to visualize the phenomenon of undamped vibration through animated frames, as shown in Figs $6(\mathrm{a})-6(\mathrm{~b})$. The animation shows, that regenerative energy in the shaft, due to internal damping is dissipated through the discrete damper and the dissipative coupling between drive and the rotor shaft. If excitation frequency is more, then the shaft speed will not increase, but the slip between drive and shaft will increase due to loading of drive. 

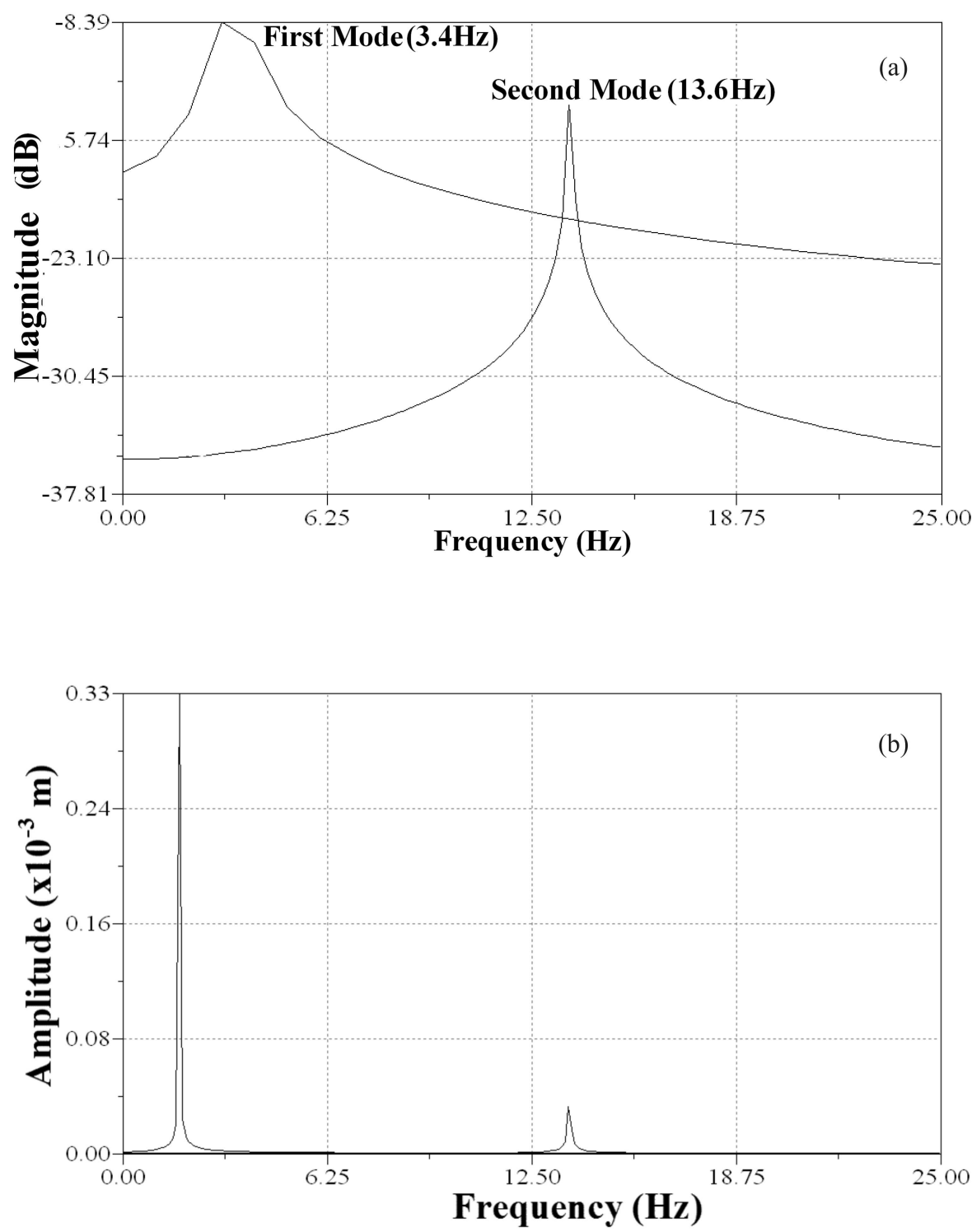

Fig. 5. (a) Superimposed frequency responses at first two undamped natural modes; (b) Amplitude of first two undamped natural modes 
External Damp. Par. $=0.020$ Internal Damp. Par. = 1.e-004

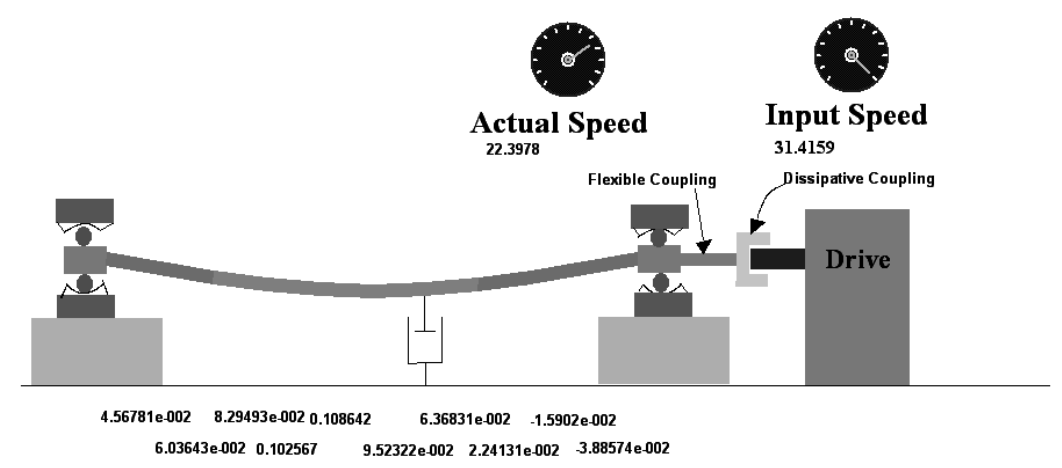

Power taken from drive in sections in $\mathbf{w}$. (a)

Internal Damp. Par. $=$ 1.e-004

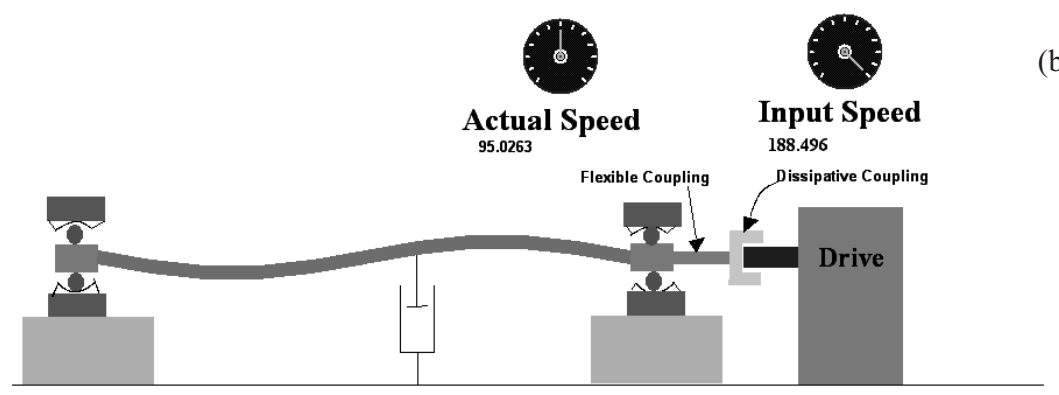

4.14411e $-003 \quad 3.42302 \mathrm{e}-002 \quad 4.88783 \mathrm{e}-003 \quad 2.00714 \mathrm{e}-002 \quad 1.4352 \mathrm{e}-002$

$\begin{array}{lllll}2.22803 \mathrm{e}-002 & 2.37352 \mathrm{e}-002 & 4.86697 \mathrm{e}-003 & 2.38073 \mathrm{e}-002 & 2.56793 \mathrm{e}-003\end{array}$

Power taken from drive in sections in $\mathbf{w}$.

Fig. 6. (a) First mode of vibration of rotor shaft with in- span concentrated external damping $R_{a}=0.020, \mu_{i}=1 e-4$, input speed $=31.41 \mathrm{rad} / \mathrm{s}$ and actual speed $=$ $22.392 \mathrm{rad} / \mathrm{s}$; (b) Second mode of vibration of rotor shaft with in- span concentrated external damping $R_{a}=6.00, \mu_{i}=1 e-4$, input speed $=188.496 \mathrm{rad} / \mathrm{s}$ and actual speed $=95.490 \mathrm{rad} / \mathrm{s}$ 


\section{Conclusions}

It has been demonstrated, that in-span discrete external damping as an isolated damper can be included in the extended Lagrangian-Hamiltonian formulation, that permits a general formulation of the dissipation effects in this new extension. The theory is further used to illustrate the significance of complex modes in vibrations of rotating shafts, which has been taken as a case study. An interesting phenomenon of limiting dynamics of a rotor shaft with in-span external damper through a dissipative coupling has been obtained. The dynamic behaviour has been analysed through extended LagrangianHamiltonain formulation for fields. The case study has been analysed theoretically and numerically. The study has further examined the various aspects of limiting dynamics of the rotor shaft and validated through simulation results. Further, the study demonstrated, that the regenerative energy in the shaft, due to elasticity/stiffness is dissipated partially through in-span external damper and the dissipative coupling. Limiting dynamics basically occurred due to the balance of power imported by internal damping from the shaft spin and dissipation of power by in-span external isolated discrete damper. Some portion of the energy has also been dissipated in the coupling and a part of action of internal damping, which acted as an external damping. The animation frames of the system have depicted the entrainment phenomenon of the whirl speed at different natural frequencies.

This new extension of Lagrangian-Hamiltonian formulation may be further used to illustrate the damped modes in vibration of cables and rods. Moreover, the concepts can also be employed to analyze the cracked beams and structures through this new extension.

\section{Nomenclature:}

$A_{n}=$ Amplitude of $n^{\text {th }}$ mode of the rotor

$E I=$ Rigidity of the continuous rotor

$H^{*}=$ Umbra-Hamiltonian of the system

$I_{d}=$ Rotary inertia of the rotor

$L_{\text {beam }}=$ Length of beam

$N_{\text {elem }}=$ Number of elements

$D_{i}=$ Internal Diameter

$D_{o}=$ External Diameter 
$\rho=$ Material density

$\mu_{i}=$ Internal Damping Coefficient

$R_{a}=$ Discrete External Damping Coefficient

$\dot{\theta}(t)=$ Shaft Spinning Speed

$R_{c}=$ Damping coefficient of dissipative coupling

$V=$ Infinitesimal generator of rotational SO (2) group

$V_{t}=$ Real time component of infinitesimal generator

$V_{\eta}=$ Umbra time component of infinitesimal generator

$n=$ Mode number

$p(\eta)=$ Umbra-time momentum

$p(t=$ Real-time momentum

$q(t)=$ Generalized displacement in real time

$q(\eta)=$ Generalized displacement in umbra-time

$\dot{q}(t)=$ Generalized velocity in real time

$\dot{q}(\eta)=$ Generalized velocity in umbra-time

$x_{i}()=$ Linear displacements in real time or umbra-time, where $i=1, \ldots, n$

$\dot{x}_{i}()=$ Linear velocity in real time or umbra-time, where $i=1, \ldots, n$

$t=$ Real-time in s.

$\Omega=$ Excitation frequency in $\mathrm{rad} / \mathrm{s}$.

$\Omega_{n}=$ Natural frequency of the rotor shaft in $\mathrm{rad} / \mathrm{s}$.

$\eta=$ Umbra-time in s.

$\omega=$ constant angular velocity

() = Angular displacement in umbra-time or real time in rad.

$\dot{\theta}()=$ Angular velocity of the shaft in umbra-time or real time in $\mathrm{rad} / \mathrm{s}$.

$L=$ Umbra-Lagrangian density

$\rho=$ Mass density of rotor shaft

$\mu_{a}=$ External damping of the beam 
$\mu_{i}=$ Internal damping of the beam

$\gamma^{*}=$ Damping ratio

$u_{i}(t)=$ Real displacement coordinates of beam

$u_{i}(\eta)=$ Umbra- displacement coordinates of beam

$H=$ Umbra-Hamiltonian density

$H_{i}, H_{e}=$ Interior and exterior umbra-Hamiltonian density.

Appendix: Umbra-Lagrangian density and generalized variational formulation

The umbra-Lagrangian density is a function, defined on an extended manifold comprising of real time, umbra and real displacements and velocities, and up to second derivatives of real and umbra displacement with respect to space coordinates $x$, which may be written as follows:

$$
L=f\left(\begin{array}{l}
t, x, U(t, x, a), V(t, x, a), \dot{U}(t, x, a), \dot{V}(t, x, a), \partial_{x} U(t, x, a) \\
\partial_{x} \dot{\partial}_{x} V(t, x, a), U(t, x, a), \partial_{x} \dot{V}(t, x, a), \partial_{x^{2}} U(t, x, a), \partial_{x^{2}} V(t, x, a), \\
U(t, x, b), V(t, x, b), \dot{U}(t, x, b), \dot{V}(t, x, b), \partial_{x} U(t, x, b), \partial_{x} V(t, x, b), \\
\partial_{x} \dot{U}(t, x, b), \partial_{x} \dot{V}(t, x, b), \partial_{x^{2}} U(t, x, b), \partial_{x^{2}} V(t, x, b)
\end{array}\right)
$$

The Lagrangian density may be written as: $L(t, x, M(t, x, a), U(t, x, b)$, $V(t, x, b), \dot{U}(t, x, b), \dot{V}(t, x, b), \ldots)$, where $a$ and $b$ are two members of the same homotopic family [29], $M(t, x, a)$ is the modulatory part and may be represented as:

$$
M(t, x, a) \approx\left(\begin{array}{l}
U(t, x, a), V(t, x, a), \dot{U}(t, x, a) \dot{V}(t, x, a), \partial_{x} U(t, x, a), \\
\partial_{x} V(t, x, a), \partial_{x} \dot{U}(t, x, a), \partial_{x} \dot{V}(t, x, a), \partial_{x}^{2} U(t, x, a), \\
\partial_{x}^{2} V(t, x, a)
\end{array}\right) .
$$

Now the action integral may be expressed as:

$$
I=\int_{t_{0}}^{t_{1}} \int_{x_{0}}^{x_{1}} L(.) d t d x
$$

This action integral will be subjected to recursive minimization. The present discussion is illustrated, using a one-dimensional continuous system. For a clear exposition, the variations in variables may be represented as follows:

$$
\begin{gathered}
U^{*}(t, x, b)=U(t, x, b)+\varepsilon \hat{U}(t, x, b), \quad \partial_{x} U^{*}(t, x, b)=\partial_{x} U(t, x, b)+\varepsilon \partial_{x} \hat{U}(t, x, b), \\
V^{*}(t, x, b)=V(t, x, b)+\varepsilon \hat{V}(t, x, b), \quad \partial_{x} V^{*}(t, x, b)=\partial_{x} V(t, x, b)+\varepsilon \partial_{x} \hat{V}(t, x, b), \\
\partial_{(t, b)} U^{*}(t, x, b)=\partial_{(t, b)} U(t, x, b)+\varepsilon \partial_{(t, b)} \hat{U}(t, x, b),
\end{gathered}
$$




$$
\begin{gathered}
\partial_{(t, b)} V^{*}(t, x, b)=\partial_{(t, b)} V(t, x, b)+\varepsilon \partial_{(t, b)} \hat{V}(t, x, b), \\
\partial_{x^{2}} U^{*}(t, x, b)=\partial_{x^{2}} U(t, x, b)+\varepsilon \partial_{x^{2}} \hat{U}(t, x, b), \\
\partial_{x^{2}} V^{*}(t, x, b)=\partial_{x^{2}} V(t, x, b)+\varepsilon \partial_{x^{2}} \hat{V}(t, x, b),
\end{gathered}
$$

It is required to propose a fixed end-points perturbation scheme on the trajectorial function $(U(t, x, b), \ldots)$, relating to the modulatory function $M(t, x, a)$ and considering path homotopic loops, based at origins as $J:\left[t_{1}, t_{2}\right] \times[0,1] \rightarrow Y$, such that $J\left(t_{1}, \xi\right)=0$ and $J\left(t_{2}, \xi\right)=0$ with $\xi \in[0,1]$, the action integral with one-dimensional variational problem, may be expressed as:

$$
I(\varepsilon)=\int_{t_{o}}^{t_{1}} \int_{x_{o}}^{x_{1}} L\left(t, x, M(t, x, a), U^{*}(t, x, b), V^{*}(t, x, b), \ldots\right) d t d x .
$$

Extremization of the action integral implies $\delta I(\varepsilon)_{\varepsilon=0}=0$, which leads to $\left.\nabla_{\varepsilon} I(\varepsilon)\right|_{\varepsilon=0}=0$. The condition of extremality results into umbra-Lagrange's field equations and boundary conditions at two ends. Now for detailed proof of umbraLagrange's field equation, it is possible to use concise notations as:

$$
\begin{gathered}
U(t, x, b)=U_{1}(t, x, b), \quad V(t, x, b)=U_{2}(t, x, b), \quad \partial_{x} U_{i}(t, x, b)=U_{i, x}(t, x, b), \\
\text { and } \partial_{x^{2}} U_{i}(t, x, b)=U_{i, x^{2}}(t, x, b),
\end{gathered}
$$

where $i=1,2$. However, in the above notations, the cognizance of the variance of the vector field is not taken. Let us consider a one-dimensional continuous system, defined over the closed domain $x_{0} \leq x \leq x_{1}$ and, $t_{1}$ and $t_{2}$ are the times, at which configurations of the system are specified and $\delta$ is the variation. On adopting such notational changes, variations in the action integral may be written as:

$$
\delta I=\varepsilon \int_{t_{0}}^{t_{1}} \int_{x_{0}}^{x_{1}} \sum_{i=1}^{2}\left\{\begin{array}{l}
\frac{\partial L}{\partial U_{i}(t, x, b)} \hat{U}_{i}(t, x, b)+\frac{\partial L}{\partial \dot{U}_{i}(t, x, b)} \dot{\hat{U}}_{i}(t, x, b) \\
+\frac{\partial L}{\partial \dot{U}_{i, x}(t, x, b)} \dot{\hat{U}}_{i, x}(t, x, b) \\
+\frac{\partial L}{\partial U_{i, x}(t, x, b)} \hat{U}_{i, x}(t, x, b) \\
+\frac{\partial L}{\partial U_{i, x^{2}}(t, x, b)} \hat{U}_{i, x^{2}}(t, x, b)
\end{array}\right\} \partial t \partial x=0
$$


Effects of Discrete Damping on the Dynamic Behaviour ...

Through integration by parts of Eq. (A5), one obtains:

$$
\delta I=\varepsilon \int_{t_{0}}^{t_{1}} \int_{x_{0}}^{x_{1}} \sum_{i=1}^{2}\left[\begin{array}{l}
\left\{\begin{array}{l}
\frac{\partial L}{\partial U_{i}(t, x, b)}-\frac{\partial}{\partial t} \frac{\partial L}{\partial \dot{U}_{i}(t, x, b)} \\
-\frac{\partial}{\partial x}\left(\frac{\partial L}{\partial U_{i, x}(t, x, b)}\right)
\end{array}\right\} \hat{U}_{i}(t, x, b) \\
+\frac{\partial}{\partial t \partial x}\left(\frac{\partial L}{\partial \dot{U}_{i, x}(t, x, b)}\right) \hat{U}_{i}(t, x, b) \\
+\frac{\partial}{\partial x^{2}}\left(\frac{\partial L}{\partial U_{i, x^{2}}(t, x, b)}\right) \hat{U}_{i}(t, x, b)
\end{array}\right] d t d x
$$

$$
+\int_{t_{0}}^{t_{1}} \sum_{i=1}^{2}\left[\begin{array}{l}
\left.\frac{\partial L}{\partial U_{i, x}(t, x, b)} \hat{U}_{i}(t, x, b)\right|_{x_{0}} ^{x_{1}} \\
-\left.\frac{\partial}{\partial t}\left(\frac{\partial L}{\partial \dot{U}_{i, x}(t, x, b)}\right) \hat{U}_{i}(t, x, b)\right|_{x_{0}} ^{x_{1}} \\
+\left.\frac{\partial L}{\partial U_{i, x^{2}}(t, x, b)} \hat{U}(t, x, b)\right|_{x_{0}} ^{x_{1}} \\
-\left.\frac{\partial}{\partial x}\left(\frac{\partial L}{\partial U_{i, x^{2}}(t, x, b)} \hat{U}_{i}(t, x, b)\right)\right|_{x_{0}} ^{x_{1}}
\end{array}\right] d t=0 .
$$

Considering the following notations as $t \times a=t, t \times b=\eta$ with $U_{i}(t, x, b)=$ $u_{i}(\eta, x)$ and $U_{i}(t, x, a)=u_{i}(t, x)$, where $i=1,2$, the first part of the Eq. (A6) gives umbra-Lagrange's field equation for one-dimensional continuous systems, when equated to zero. Now re-arranging first part in $\eta-t$ form, umbra field equations may be written after taking the limit $\eta \rightarrow t$ as:

$$
\begin{gathered}
\frac{\partial}{\partial t}\left\{\operatorname{Lim}_{\eta \rightarrow t}\left(\frac{\partial L}{\partial \dot{u}_{i}(\eta, x)}-\frac{\partial}{\partial x}\left(\frac{\partial L}{\partial \dot{u}_{i, x}(\eta, x)}\right)\right)\right\} \\
-\operatorname{Lim}_{\eta \rightarrow t}\left(\begin{array}{c}
\frac{\partial L}{\partial u_{i}(\eta, x)}-\frac{\partial}{\partial x}\left(\frac{\partial L}{\partial u_{i, x}(\eta, x)}\right) \\
+\frac{\partial}{\partial x^{2}}\left(\frac{\partial L}{\partial u_{i, x^{2}}(\eta, x)}\right)
\end{array}\right)=0,
\end{gathered}
$$

where $i=1,2$, and $u_{i, x}=\partial u_{i} / \partial x$.

The second part of Eq. (A6) gives the boundary conditions, when equated to zero, which reflects the physical (geometric) conditions at the two ends and may be expressed as:

$\left.(A 8 a) \operatorname{Lim}_{\eta \rightarrow t}\left[\frac{\partial L}{\partial u_{i, x}(\eta, x)}-\frac{\partial}{\partial x}\left(\frac{\partial L}{\partial u_{i, x^{2}}(\eta, x)}\right)-\frac{\partial}{\partial \eta}\left(\frac{\partial L}{\partial \dot{u}_{i, x}(\eta, x)}\right)\right] \hat{u}_{i}(\eta, x)\right|_{x_{0}} ^{x_{1}}=0$ 


$$
\left.\operatorname{Lim}_{\eta \rightarrow t} \frac{\partial L}{\partial u_{i, x^{2}}(\eta, x)} \hat{u}_{i, x}(\eta, x)\right|_{x_{0}} ^{x_{1}}=0
$$

where $i=1,2$. There exist the following possibilities in the boundary conditions. Either $\operatorname{Lim}_{\eta \rightarrow t}\left[\frac{\partial L}{\partial u_{i, x}(\eta, x)}-\frac{\partial}{\partial x}\left(\frac{\partial L}{\partial u_{i, x^{2}}(\eta, x)}\right)-\frac{\partial}{\partial \eta}\left(\frac{\partial L}{\partial \dot{u}_{i, x}(\eta, x)}\right)\right]=0$ or $u_{i}(t, x)=$ 0 , and either $\operatorname{Lim}_{\eta \rightarrow t} \frac{\partial L}{\partial u_{i, x^{2}}(\eta, x)}=0$ or $u_{i, x}(t, x)=0$, at either of the ends $x=x_{0}$ and $x=x_{1}$. Equation (A7) is the dynamical equations, governing the motion of a onedimensional continuous system (or field) with generalized co-ordinates. The solution of this equation, combined with the appropriate boundary conditions, given by Eqs. (A8a) and (A8b), specify the true motion of such continuous system, the motion that ensures the action integral of Eq. (A4) to be extreme.

\section{REFERENCES}

[1] Boyer, T. H. Continuous Symmetries and Conserved Currents. Annals of Physics, 42 (1967), No. 3, 445-466.

[2] Sarlet, W., F. Cantrijn. Generalizations of Noether's Theorem in Classical Mechanics. SIAM Rev., 23 (1981), No. 3, 467

[3] Katzin, G. H., J. Levine. A Gauge Invariant Formulation of Time-dependent Dynamical Symmetry Mappings and Associated Constants of Motion for Lagrangian Particle Mechanics. Int. J. Math. Phys., 17 (1976). No. 7, 1345.

[4] Damianou, P. A., C. Sophocleous. Classification of Noether Symmetries for Lagrangians with Three Degree of Freedom. Nonlinear Dynamics, 36 (2004), No. 1, 3-18.

[5] MukherJeE, A. Junction Structures of Bondgraph Theory from Analytical Mechanics Viewpoint, Proc. of CISS- $1^{\text {st }}$ Conference of International Simulation Societies, Switzerland, Zurich, 1994, 661-664.

[6] Brown, F. T. Engineering System Dynamics, 2-nd ed, CRC, Taylor \& Francis, 2007.

[7] Mukherjee, A., V. Rastogi, A. DasGupta. A Methodology for Finding Invariants of Motion for Asymmetric Systems with Gauge-transformed Umbra Lagrangian Generated by Bond Graphs. Simulation, 82 (2006), No. 4, 207-226.

[8] Mukherjee, A., V. Rastogi, A. DasGupta. A Study of a Bi-Symmetric Electro-mechanical System through Umbra Lagrangian Generated by Bond Graphs, and Noether's Theorem. Simulation, 83 (2007), No. 9, 611-630.

[9] Mukherjee, A., V. Rastogi, A. DasGupta. Extension of LagrangianHamiltonian Mechanics for Continuous Systems-Investigation of Dynamics of a One Dimensional Internally Damped Rotor driven through a Dissipative Coupling. Nonlinear Dynamics, 58 (2009), No. 1, 107-127. 
[10] Mukherjee, A., V. Rastogi, A. DasGupta. Revisiting Umbra-LagrangianHamiltonian Mechanics: Its Variational Foundation and Extension of Noether's Theorem and Poincaré-Cartan Integral. International Journal of Non-Linear Mechanics, 46 (2011), No. 5, 745-757.

[11] Rastogi, V. Extension of Lagrangian-Hamiltonian Mechanics, Study of Symmetries and Invariants, Ph. D. thesis, Kharagpur, Indian Institute of Technology, 2005.

[12] Bogoliubov, N. Perturbation Theory in Nonlinear Mechanics. Collection of Works of Building Mechanics Institute, 4 (1950), 9-34, (In Russian).

[13] Meirovitch, L. Elements of Vibration Analysis, McGraw-Hill Book Company, 1984.

[14] Krämer, E. Dynamics of Rotors and Foundations, Springer-Verlag, 1993.

[15] Kimball, A. L. Internal Friction Theory of Shaft Whirling. General Electrical Review, 27 (1924), 244.

[16] Dimentberg, M. Flexural Vibrations of Rotating Shafts, Butter Worth, London, England, 1961.

[17] Gunter, E. J. Rotor-Bearing Stability, Proceeding of the First Turbo Machinery Symposium, 1972, 119-141.

[18] Ehrich, F. E. Shaft Whirl Induced by Rotor Internal Damping. Journal of Applied Mechanics, 31 (1964), 279-282.

[19] Crandall, S. H. The Role of Damping in Vibration Theory, Journal of Sound and Vibration, 11 (1970), 3-18.

[20] Zorzi, E. S., H. D. Nelson. Finite Element Simulation of Rotor Bearing Systems with Internal Damping. Journal of Engineering Power, ASME trans., 99 (1977), 71-76.

[21] Melanson, J., J. W. Zu. Free Vibration and Stability Analysis of Internally Damped Rotating Shafts with General Boundary Conditions. J. Vibration Acoustics, ASME Trans., 120 (1998), 776-783.

[22] Genta, G. On a Persistent Misunderstanding of the Role of Hysteretic Damping in Rotor Dynamics. J. Vibration Acoustics, ASME Trans., 126 (2004), 459-461.

[23] Vance, J. M., J. Lee. Stability of High Speed Rotors with Internal Friction. J. of Engineering for Industry, Trans. ASME, Series B, 96 (1974), No. 3, 960-966.

[24] Szorc, T. On the Discrete Continuous Modelling of Rotor System for the Analysis of Coupled Lateral Vibration. Internaltional Journal of Rotating Machinery, 6 (2000), No. 2, 135-149.

[25] Jivkov, V., E. Zahariev. Stability and Non-Stationary Vibrations of Rotor in Elasto-Viscous Field, Mechanics Based Design of Structures and Machines. Taylor \& Francis Group, 42 (2014), No. 1, $35-55$.

[26] Krenk, S. Complex Modes and Frequencies in Damped Structural Vibrations. Journal of Sound and Vibration, 270 (2004), 981-996.

[27] Noether, E. Invariante Variationsprobleme. Ges. Wiss. Gottington, 2 (1918), 235. 
[28] Mukherjee, A., R. Karmakar, A. Samantaray. Modelling and Simulation of Engineering System through Bond Graph, New Delhi, Narosa Publishing House, reprinted by CRC press for North America and by Alpha Science for Europe, 2000 .

[29] Karnopp, D. C., R. C. Resenberg, D. L. Margolis. System Dynamics: A Unified Approach, USA, John-Wiley and Sons Inc., 1990.

[30] Mukherjee, A., A. K. Samantaray. SYMBOLS-2000 User's Manual, India, Kharagpur, High-Tech Consultants, STEP Indian Institute of Technology, 2000.

[31] Internet URL://www. Symbols2000.com, 2000.

[32] Arnold, V. I. Mathematical Methods of Classical Mechanics, New York Heidelberg-Berlin, Springer-Verlag, 1974, 88.

[33] Olver, P. Application of Lie Groups to Differential Equations, Springer-Verlag, 1986.

[34] Hassani, S. Mathematical Physics, New York-Heidelberg-Berlin, SpringerVerlag, 1999, 936.

[35] Munkres, J. R. Topology: A First Course, New Delhi, Prentice Hall of India Private Limited, 1994. 